\title{
Properties of the solutions to the Monge-Ampère equation
}

\author{
Liliana Forzani ${ }^{\mathrm{a}, \mathrm{b}}$, Diego Maldonado ${ }^{\mathrm{c}, *}$ \\ ${ }^{a}$ Unl-Imal-Conicet, Argentina \\ ${ }^{\mathrm{b}}$ School of Statistics, University of Minnesota, 313 Ford Hall, 224 Church Street, Minneapolis, \\ $M N-55455$, USA \\ ${ }^{\mathrm{c}}$ Department of Mathematics, University of Kansas, 1460 Jayhawk Blvd., 405 Snow Hall, \\ KS-66045, USA
}

Received 16 September 2003; accepted 10 March 2004

\begin{abstract}
We consider solutions to the equation $\operatorname{det} D^{2} \varphi=\mu$ when $\mu$ has a doubling property. We prove new geometric characterizations for this doubling property (by means of the so-called engulfing property) and deduce the quantitative behaviour of $\varphi$. Also, a constructive approach to the celebrated $C^{1, \beta}$-estimates proved by L. Caffarelli is presented, settling one of the open questions posed by Villani (Amer. Math. Soc. 58 (2003)).
\end{abstract}

(C) 2004 Elsevier Ltd. All rights reserved.

MSC: primary 35J60, 35D10; secondary 26B25

Keywords: Monge-Ampère equation; Monge-Ampère measure; Sections of convex functions

\section{Introduction}

Let $\varphi: \mathbb{R}^{n} \rightarrow \mathbb{R}$ be a convex function and let $\partial \varphi$ denote its normal mapping (or sub-differential). The Monge-Ampère measure $\mu_{\varphi}$ associated to $\varphi$ is defined on any Borel set $E$ by

$$
\mu_{\varphi}(E)=|\partial \varphi(E)|,
$$

where $|\cdot|$ stands for Lebesgue measure. For $x \in \mathbb{R}^{n}, p \in \partial \varphi(x)$ and $t>0$, a section of $\varphi$ centered in $x$ at height $t$ is the open convex set

$$
S_{\varphi}(x, p, t)=\left\{y \in \mathbb{R}^{n}: \varphi(y)<\varphi(x)+p \cdot(y-x)+t\right\} .
$$

\footnotetext{
* Corresponding author. Tel.: +1-785-864-5269; fax: +1-785-864-5255.

E-mail addresses: lforzani@math.unl.edu.ar (L. Forzani), maldonado@math.ukans.edu (D. Maldonado).
} 
Throughout this paper, we shall only consider functions $\varphi$ whose sections are bounded sets. Geometrically, this means that the graph of $\varphi$ does not contain half-lines. If $\varphi$ is differentiable, then we identify $\partial \varphi(x)$ and $\nabla \varphi(x)$. In this case, we just write $S_{\varphi}(x, t)$ for the sections.

If we consider the archetypal convex function $\varphi_{0}(x)=\frac{1}{2}|x|^{2}$, then the Monge-Ampère measure associated to $\varphi_{0}$ is exactly Lebesgue measure, and for $x \in \mathbb{R}^{n}$ and $t>0$

$$
S_{\varphi_{0}}(x, t)=B(x, \sqrt{2 t}) .
$$

Hence, the family of sections of $\varphi_{0}$ consists of the usual balls in $\mathbb{R}^{n}$. Many conditions on a general $\varphi$ have been proposed in order to preserve the harmony between measure theory and geometry enjoyed in the case of $\varphi_{0}$. The study of these properties began with the fundamental papers of Caffarelli [2,3], Caffarelli and Gutiérrez [4,5]; and was continued by Gutiérrez and Huang [9], and the Forzani and Maldonado [6,7]. Some of these conditions are imposed on the sections of $\varphi$. For instance, we say that the sections satisfy the engulfing property if there exists a $K>1$ such that for every section $S_{\varphi}(x, p, t)$ it holds:

$$
y \in S_{\varphi}(x, p, t) \Rightarrow S_{\varphi}(x, p, t) \subset S_{\varphi}(y, q, K t)
$$

for all $q \in \partial \varphi(y)$. Also, some of the conditions are imposed on the measure $\mu_{\varphi}$, for instance, we say that $\mu_{\varphi}$ satisfies the (DC)-doubling property if there exist constants $C>0$ and $0<\alpha<1$ such that for all sections $S_{\varphi}(x, p, t)$, we have

$$
\mu_{\varphi}\left(S_{\varphi}(x, p, t)\right) \leqslant C \mu_{\varphi}\left(\alpha S_{\varphi}(x, p, t)\right),
$$

where $\alpha S_{\varphi}(x, p, t)$ denotes $\alpha$-dilation with respect to the center of mass of $S_{\varphi}(x, p, t)$. This property of $\mu_{\varphi}$ plays a remarkable role in the regularity theory for solutions to the linearized Monge-Ampère equation, see [5,8]. In [9], Gutiérrez and Huang proved that the (DC)-doubling property for $\mu_{\varphi}$ implies the engulfing property for the sections of $\varphi$. On the other hand, in [6] the authors proved the converse of that result. The interplay between geometry and measure theory can be summarized in the following theorem (see $[6,9]$ for these and other equivalent conditions).

Theorem 1. Let $S_{\varphi}(x, p, t), x \in \mathbb{R}^{n}, p \in \partial \varphi(x), t>0$ be the bounded sections of a convex function $\varphi$. Then the following are equivalent:

(i) The sections of $\varphi$ satisfy the engulfing property.

(ii) The measure $\mu_{\varphi}$ satisfies the (DC)-doubling property.

(iii) The Monge-Ampère measure $\mu_{\varphi}$ satisfies

$$
c t^{n} \leqslant\left|S_{\varphi}(x, p, t)\right| \mu_{\varphi}\left(S_{\varphi}(x, p, t)\right) \leqslant t^{n},
$$

for all sections $S_{\varphi}(x, p, t)$ and some positive constants $c, C$.

Moreover, the (DC)-doubling property implies two important properties for $\varphi$ when its sections are bounded sets: $\varphi$ is strictly convex, and $\varphi \in C^{1, \beta}(Q)$, where $Q \subset \mathbb{R}^{n}$ is any compact set and $\beta$ depends on $Q$. These results were first proved by Caffarelli [3]. See also Gutiérrez' book [8] for a comprehensive exposition of these and other results related to the Monge-Ampère equation. 
On the other hand, if $\varphi: \mathbb{R}^{n} \rightarrow \mathbb{R}$ is strictly convex and differentiable, we set

$$
\rho_{\varphi}(x, y)=\inf \left\{r: y \in S_{\varphi}(x, r), x \in S_{\varphi}(y, r)\right\}
$$

and

$$
d_{\varphi}(x, y)=(\nabla \varphi(x)-\nabla \varphi(y)) \cdot(x-y),
$$

then it is immediate to check that

$$
\rho_{\varphi}(x, y) \leqslant d_{\varphi}(x, y) \leqslant 2 \rho_{\varphi}(x, y),
$$

for every $x, y \in \mathbb{R}^{n}$. In [1] Aimar et al. proved that: if the sections of $\varphi$ satisfy the engulfing property with constant $K$, then $\rho_{\varphi}$ (as much as $d_{\varphi}$ ) is a quasi-distance on $\mathbb{R}^{n}$ whose balls are topologically equivalent to the sections of $\varphi$, that is, there exist positive constants $0<\delta_{1}<1<\delta_{2}$, depending only on $K$, such that

$$
S_{\varphi}\left(x, \delta_{1} t\right) \subset B_{\rho_{\varphi}}(x, t) \subset S_{\varphi}\left(x, \delta_{2} t\right),
$$

for every $x \in \mathbb{R}^{n}$ and $t>0$. Moreover, the quasi-triangular constant of $\rho_{\varphi}$ depends only on $K$. Conversely, if $\rho$ is any quasi-distance on $\mathbb{R}^{n}$ whose balls are topologically equivalent to the sections of $\varphi$, then the sections of $\varphi$ have the engulfing property; this is just due to the quasi-triangular inequality for $\rho$. Also, since the (DC)-doubling property of $\mu_{\varphi}$ implies another doubling condition of $\mu_{\varphi}$ on the sections, now with respect to the parameter $t$ (see $[8,9]$ ), we have that the engulfing property turns $\left(\mathbb{R}^{n}, d_{\varphi}, \mu_{\varphi}\right)$ into a space of homogeneous type. Consequently, the real analysis (types of the Hardy-Littlewood maximal function, Calderón-Zygmund decomposition, BMO, Hardy spaces, singular integrals, Muckenhoupt's classes, etc.) with respect to $\mu_{\varphi}$ and the sections of $\varphi$ follows in a standard way. This is another important application of convex functions satisfying the engulfing property.

To cite some other recent applications of these ideas, let us mention that in [7], the authors proved the following characterization for the engulfing property in dimension 1 which, in turn, is useful to characterize all doubling measures and quasi-symmetric mappings on $\mathbb{R}$.

Theorem 2. Let $\varphi: \mathbb{R} \rightarrow \mathbb{R}$ be a strictly convex differentiable function. The following are equivalent:

(i) (Engulfing property of the sections of $\varphi$.) There exists a constant $K>1$ such that if $x \in S_{\varphi}(y, t)$ then

$$
S_{\varphi}(y, t) \subset S_{\varphi}(x, K t),
$$

for every $x, y \in \mathbb{R}$ and $t>0$.

(ii) There exists a constant $K^{\prime}>1$ such that if $x, y \in \mathbb{R}$ and $t>0$ verify $x \in S_{\varphi}(y, t)$, then $y \in S_{\varphi}\left(x, K^{\prime} t\right)$.

(iii) There exists a constant $K^{\prime \prime}>1$ such that for every $x, y \in \mathbb{R}$

$$
\begin{aligned}
& \frac{K^{\prime \prime}+1}{K^{\prime \prime}}\left(\varphi(y)-\varphi(x)-\varphi^{\prime}(x)(y-x)\right) \\
& \quad \leqslant\left(\varphi^{\prime}(x)-\varphi^{\prime}(y)\right)(x-y) \\
& \quad \leqslant\left(K^{\prime \prime}+1\right)\left(\varphi(y)-\varphi(x)-\varphi^{\prime}(x)(y-x)\right) .
\end{aligned}
$$


Let us denote by $\operatorname{Eng}(n, K)$ the set of all convex functions $\varphi: \mathbb{R}^{n} \rightarrow \mathbb{R}$ whose bounded sections satisfy the engulfing property with constant $K$. Let us also define

$$
\operatorname{Eng}(n)=\bigcup_{K>1} \operatorname{Eng}(n, K)
$$

and

$$
\operatorname{Eng}_{0}(n)=\bigcup_{K>1} \operatorname{Eng}_{0}(n, K),
$$

where $\operatorname{Eng}_{0}(n, K)=\{\varphi \in \operatorname{Eng}(n, K): \varphi(0)=0, \nabla \varphi(0)=0\}$.

The purpose of this paper is to exhibit new characterizations for the engulfing property and to describe the quantitative behaviour of functions in $\operatorname{Eng}(n)$. We do this by means of a multi-dimensional version of Theorem 2 . Then, several properties of functions in $\operatorname{Eng}(n)$ are deduced. We also stress the importance of convex conjugate functions, in particular, we prove that $\operatorname{Eng}(n)$ is invariant under conjugation. The last part of the paper is devoted to the constructive estimates of Caffarelli's $C^{1, \beta}$-theory.

\section{Examples of functions in $\operatorname{Eng}(n)$}

Let $\varphi: \mathbb{R}^{n} \rightarrow \mathbb{R}$ be a strictly convex differentiable function.

(i) If $\operatorname{det} D^{2} \varphi=p$, where $p$ is a polynomial, then $\varphi \in \operatorname{Eng}(n, K)$ for some $K$ depending only on the degree of $p$ (in particular, $K$ does not depend on the coefficients of $p$ ), see [8, p. 52].

(ii) If $\mu_{\varphi}$ verifies the $\mu_{\infty}$ property, i.e., given $\delta_{1} \in(0,1)$, there exists $\delta_{2} \in(0,1)$ such that for every section $S=S_{\varphi}(x, t)$ and every measurable set $E \subset S$,

$$
\frac{|E|}{|S|}<\delta_{2} \Rightarrow \frac{\mu_{\varphi}(E)}{\mu_{\varphi}(S)}<\delta_{1}
$$

then $\varphi \in \operatorname{Eng}(n)$. To see how $\mu_{\infty}$ implies the (DC)-doubling condition, given $\delta_{1} \in(0,1)$, pick $\alpha \in(0,1)$ such that

$$
\frac{|S-\alpha S|}{|S|}=1-\alpha^{n}<\delta_{2}
$$

then

$$
\frac{\mu_{\varphi}(S-\alpha S)}{\mu_{\varphi}(S)}<\delta_{1}
$$

and the (DC)-doubling property follows with $C=1 /\left(1-\delta_{1}\right)$. By Theorem 1 , we get $\varphi \in \operatorname{Eng}(n)$. This $\mu_{\infty}$ property plays an important role in the proof of Harnack's inequality for non-negative solutions to the linearized Monge-Ampère equation, see [5].

(iii) If $\varphi \in C^{2}\left(\mathbb{R}^{n}\right)$ and there exist constants $\lambda, \Lambda>0$ such that

$$
\lambda \leqslant \operatorname{det} D^{2} \varphi \leqslant \Lambda,
$$

then $\varphi \in \operatorname{Eng}(n)$. This follows from the fact that in this case $\mu_{\varphi}$ clearly verifies the $\mu_{\infty}$ property. Actually, the same is true if we only ask (2.2) to hold in the Aleksandrov sense. 
(iv) If $n=1$ and $\varphi(x)=|x|^{p}$ with $p>1$, then $\varphi \in \operatorname{Eng(1).~In~general,~if~} \mu$ is a doubling measure on $\mathbb{R}$, then $\varphi_{\mu}(x)=\int_{0}^{x} \int_{0}^{t} \mathrm{~d} \mu \mathrm{d} t$ belongs to $\operatorname{Eng}_{0}(1)$, see [7].

\section{Some immediate properties of $\operatorname{Eng}(n)$}

Lemma 3. Let $\varphi$ be in $\operatorname{Eng}(n, K)$.

(i) If $\lambda>0$, then $\lambda \varphi \in \operatorname{Eng}(n, K)$.

(ii) If $\psi \in \operatorname{Eng}\left(n, K^{\prime}\right)$, then $\varphi+\psi \in \operatorname{Eng}\left(n, 2\left(K \vee K^{\prime}\right)\right)$.

(iii) If for $x, y \in \mathbb{R}^{n}$ we set $\varphi_{x, y}(s)=\varphi(s y+(1-s) x), s \in \mathbb{R}$, then $\varphi_{x, y} \in \operatorname{Eng}(1, K)$.

(iv) $\operatorname{Aff}\left(\mathbb{R}^{n}, \mathbb{R}^{n}\right)$ acts on $\operatorname{Eng}(n, K)$ by composition.

(v) $\operatorname{Aff}\left(\mathbb{R}^{n}, \mathbb{R}\right)$ acts on $\operatorname{Eng}(n, K)$ by addition.

Proof. In order to prove (i), we observe that given $z \in \mathbb{R}^{n}$ and $\lambda, s>0$, we have

$$
S_{\lambda \varphi}(z, s)=S_{\varphi}(z, s / \lambda)
$$

Now, if $y \in S_{\lambda \varphi}(x, t)$, then $y \in S_{\varphi}(x, t / \lambda)$. By the engulfing property of $\varphi, S_{\varphi}(x, t / \lambda) \subset$ $S_{\varphi}(y, K t / \lambda)$. And, according to (3.3), $S_{\lambda \varphi}(x, t) \subset S_{\lambda \varphi}(y, K t)$. Hence, $\lambda \varphi \in \operatorname{Eng}(n, K)$.

To prove (ii), first note that for every $z \in \mathbb{R}^{n}$ and $s>0$,

$$
S_{\varphi+\psi}(z, s) \subset S_{\varphi}(z, s) \cap S_{\psi}(z, s) \subset S_{\varphi+\psi}(z, 2 s) .
$$

In particular, the sections of $\varphi+\psi$ are bounded sets. Now, if $y \in S_{\varphi+\psi}(x, t)$ then $y \in S_{\varphi}(x, t) \cap S_{\psi}(x, t)$, which implies $S_{\varphi}(x, t) \subset S_{\varphi}(y, K t)$ and $S_{\psi}(x, t) \subset S_{\psi}\left(y, K^{\prime} t\right)$. Therefore, setting $K^{\prime \prime}=K \vee K^{\prime}$ and using (3.4), we obtain

$$
\begin{aligned}
S_{\varphi+\psi}(x, t) & \subset S_{\varphi}(x, t) \cap S_{\psi}(x, t) \subset S_{\varphi}(y, K t) \cap S_{\psi}\left(y, K^{\prime} t\right) \\
& \subset S_{\varphi}\left(y, K^{\prime \prime} t\right) \cap S_{\psi}\left(y, K^{\prime \prime} t\right) \subset S_{\varphi+\psi}\left(y, 2 K^{\prime \prime} t\right) .
\end{aligned}
$$

(iii) For $r, s \in \mathbb{R}$ and $t>0$, we have

$$
r \in S_{\varphi_{x, y}}(s, t) \Leftrightarrow r y+(1-r) x \in S_{\varphi}(s y+(1-s) x, t) .
$$

Thus, if $r \in S_{\varphi_{x, y}}(s, t)$ then, by (3.5) and the engulfing property of $\varphi$, we have $s y+$ $(1-s) x \in S_{\varphi}(r y+(1-r) x, K t)$. By (3.5) again, we have $s \in S_{\varphi_{x, y}}(r, K t)$. The engulfing property for $\varphi_{x, y}$ now follows from Theorem 2, with constant $K$ independent of $x$ and $y$.

(iv) Given $T \in G L(n, \mathbb{R})$ and $b \in \mathbb{R}^{n}$, set $\varphi_{A}=\varphi \circ A$, where $A x=T x+b$. First note that for all $u, v \in \mathbb{R}^{n}$ and $s>0$ we have

$$
u \in S_{\varphi_{A}}(v, s) \Leftrightarrow A u \in S_{\varphi}(A v, s) .
$$

Now, $y \in S_{\varphi_{A}}(x, t) \Rightarrow A y \in S_{\varphi}(A x, t)$. By the engulfing property we have $S_{\varphi}(A x, t) \subset$ $S_{\varphi}(A y, K t)$. Now, this last inclusion and (3.6) imply that $S_{\varphi_{A}}(x, t) \subset S_{\varphi_{A}}(y, K t)$, the engulfing property for $\varphi_{A}$. Finally, we use the condition $\operatorname{det} T \neq 0$ to assure the boundedness of the sections of $\varphi_{A}$. Thus, $\varphi_{A} \in \operatorname{Eng}(n, K)$. 
(v) Fix $v \in \mathbb{R}^{n}, b \in \mathbb{R}$ and define $a(x)=v \cdot x+b$. Set $\psi(x)=\phi(x)+a(x)$. It is immediate that

$$
S_{\psi}(x, t)=S_{\varphi}(x, t),
$$

for every $x \in \mathbb{R}^{n}$ and $t>0$. Thus, if $\phi \in \operatorname{Eng}(n, K)$ then $\psi \in \operatorname{Eng}(n, K)$.

\section{New characterizations for the engulfing property}

The following result is the $n$-dimensional version of Theorem 2 .

Theorem 4. Let $\varphi: \mathbb{R}^{n} \rightarrow \mathbb{R}$ be a strictly convex differentiable function. The following are equivalent:

(i) There exists a constant $K>1$ such that if $x \in S_{\varphi}(y, t)$ then

$$
S_{\varphi}(y, t) \subset S_{\varphi}(x, K t),
$$

for every $x, y \in \mathbb{R}^{n}$ and $t>0$.(Engulfing property.)

(ii) There exists a constant $K^{\prime}>1$ such that if $x, y \in \mathbb{R}^{n}$ and $t>0$ verify $x \in S_{\varphi}(y, t)$, then $y \in S_{\varphi}\left(x, K^{\prime} t\right)$.

(iii) There exists a constant $K^{\prime \prime}>1$ such that for every $x, y \in \mathbb{R}^{n}$

$$
\begin{aligned}
& \frac{K^{\prime \prime}+1}{K^{\prime \prime}}(\varphi(y)-\varphi(x)-\nabla \varphi(x) \cdot(y-x)) \\
& \quad \leqslant(\nabla \varphi(x)-\nabla \varphi(y)) \cdot(x-y) \\
& \quad \leqslant\left(K^{\prime \prime}+1\right)(\varphi(y)-\varphi(x)-\nabla \varphi(x) \cdot(y-x)) .
\end{aligned}
$$

Proof. The proof for (i) $\Rightarrow$ (ii) is obvious since $y \in S_{\varphi}(y, t)$ for every $y \in \mathbb{R}^{n}$ and $t>0$. Thus (ii) holds with $K^{\prime}=K$.

Proof of (ii) $\Rightarrow$ (iii): Given $x, y \in \mathbb{R}^{n}$ and $\varepsilon>0$, we have

$$
\begin{gathered}
\varphi(x)<\varphi(x)+\varepsilon=\varphi(y)+\nabla \varphi(y) \cdot(x-y)+\varphi(x)-\varphi(y) \\
-\nabla \varphi(y) \cdot(x-y)+\varepsilon,
\end{gathered}
$$

(note that the convexity of $\varphi$ implies $\varphi(x)-\varphi(y)-\nabla \varphi(y) \cdot(x-y) \geqslant 0$ ), this means that $x \in S_{\varphi}(y, \varphi(x)-\varphi(y)-\nabla \varphi(y) \cdot(x-y)+\varepsilon)$. By property (ii), we must have $y \in S_{\varphi}\left(x, K^{\prime}(\varphi(x)-\varphi(y)-\nabla \varphi(y) \cdot(x-y)+\varepsilon)\right)$, which means

$$
\varphi(y) \leqslant \varphi(x)+\nabla \varphi(x) \cdot(y-x)+K^{\prime} \varphi(x)-K^{\prime} \varphi(y)-K^{\prime} \nabla \varphi(y) \cdot(x-y)+K^{\prime} \varepsilon .
$$

Letting $\varepsilon$ go to 0 and summing up we get

$$
\left(K^{\prime}+1\right) \varphi(y) \leqslant\left(K^{\prime}+1\right) \varphi(x)+\left(\nabla \varphi(x)+K^{\prime} \nabla \varphi(y)\right)(y-x) .
$$

Now interchanging the roles of $x$ and $y$, we obtain

$$
\left(K^{\prime}+1\right) \varphi(x) \leqslant\left(K^{\prime}+1\right) \varphi(y)+\left(\nabla \varphi(y)+K^{\prime} \nabla \varphi(x)\right)(x-y) .
$$


From (4.8) and (4.9), we get

$$
\begin{aligned}
& \frac{1}{K^{\prime}}+1 \\
& \quad \leqslant \varphi(x) \cdot(x-y)+\frac{K^{\prime}}{K^{\prime}+1} \nabla \varphi(y)(x-y) \\
& \quad \leqslant\left(\frac{1}{K^{\prime}+1} \nabla \varphi(y)+\frac{K^{\prime}}{K^{\prime}+1} \nabla \varphi(x)\right)(x-y) .
\end{aligned}
$$

By using the first inequality in (4.10) we get

$$
\frac{1}{K^{\prime}+1}(\nabla \varphi(x)-\nabla \varphi(y)) \cdot(x-y) \leqslant \varphi(x)-\varphi(y)-\nabla \varphi(y)(x-y) .
$$

The second inequality in (4.10) yields

$$
\varphi(x)-\varphi(y)-\nabla \varphi(x) \cdot(x-y) \leqslant \frac{1}{K^{\prime}+1}(\nabla \varphi(y)-\nabla \varphi(x))(x-y),
$$

which implies

$$
\varphi(x)-\varphi(y)-\nabla \varphi(y) \cdot(x-y) \leqslant \frac{K^{\prime}}{K^{\prime}+1}(\nabla \varphi(x)-\nabla \varphi(y))(x-y) .
$$

Now (iii) follows from (4.13) and (4.11) with $K^{\prime \prime}=K^{\prime}$.

Proof of (iii) $\Rightarrow$ (ii): Suppose $x \in S_{\varphi}(y, t)$, then

$$
\varphi(x)-\varphi(y)-\varphi^{\prime}(y)(x-y)<t,
$$

now, by the second inequality in (iii), we get

$$
(\nabla \varphi(x)-\nabla \varphi(y))(x-y)=(\nabla \varphi(y)-\nabla \varphi(x)) \cdot(y-x) \leqslant\left(K^{\prime \prime}+1\right) t
$$

and by using the first inequality in (iii),

$$
\varphi(y)-\varphi(x)-\nabla \varphi(x) \cdot(y-x) \leqslant K^{\prime \prime} t .
$$

That is, $y \in S_{\varphi}\left(x, K^{\prime \prime} t\right)$; and (ii) follows with $K^{\prime}=K^{\prime \prime}$.

(ii) $\Rightarrow$ (i): Let $x \in S_{\varphi}(y, t)$. We want to prove the existence of a constant $K>1$ such that

$$
S_{\varphi}(y, t) \subset S_{\varphi}(x, K t) .
$$

Let us assume first that $\varphi(y)=0$ and $\nabla \varphi(y)=0$ (in particular, we get $\varphi \geqslant 0$ ). Consider the line going through $x$ and $z, s x+(1-s) z, s \in \mathbb{R}$, and let $s_{1}, s_{2} \in \mathbb{R}$ such that

$$
\varphi\left(s_{1} x+\left(1-s_{1}\right) z\right)=\varphi\left(s_{2} x+\left(1-s_{2}\right) z\right)=t .
$$

By the strict convexity of $\varphi$, the segment $I=\{s \in \mathbb{R}: s x+(1-s) z\} \cap S_{\varphi}(y, t)$ equals a certain section $S_{\varphi_{x, z}}(h, l)$ of $\varphi_{x, z}$ (as defined in Lemma 3 ) for some $h \in I$ and $l>0$ such that

$$
\varphi_{x, z}\left(s_{1}\right)-\varphi_{x, z}(h)-\varphi_{x, z}^{\prime}\left(s_{1}-h\right)=\varphi_{x, z}\left(s_{2}\right)-\varphi_{x, z}(h)-\varphi_{x, z}^{\prime}\left(s_{2}-h\right)=l,
$$

which implies

$$
\varphi_{x, z}\left(s_{1}\right)-\varphi_{x, z}\left(s_{2}\right)-\varphi_{x, z}^{\prime}(h)\left(s_{1}-s_{2}\right)=0,
$$


and, since $s_{1} \neq s_{2}$ and $\varphi_{x, z}\left(s_{1}\right)=\varphi_{x, z}\left(s_{2}\right)=t$, we get $\varphi_{x, z}^{\prime}(h)=0$; this equality, together with the non-negativity of $\varphi$ and (4.14), implies $l \leqslant t$. Since $x \in S_{\varphi}(y, t)$, we have $\varphi_{x, z}(1)=\varphi(x)<t$. Hence,

$$
1 \in S_{\varphi_{x, z}}(h, t) \text {. }
$$

On the other hand, since $\varphi$ verifies (ii) with constant $K^{\prime}$ then it is straightforward that $\varphi_{x, z}$ verifies (ii) in Theorem 2 with the same constant $K^{\prime}$, and by Theorem 2 we get that $\varphi_{x, z} \in \operatorname{Eng}(1, K)$, where $K$ depends only on $K^{\prime}$. Actually, we can take $K=2 K^{\prime}\left(K^{\prime}+1\right)$, see [7]. Thus, (4.15) implies

$$
S_{\varphi_{x, z}}(h, t) \subset S_{\varphi_{x, z}}(1, K t) .
$$

But, the fact that $z \in S_{\varphi}(y, t)$ can be written as $0 \in S_{\varphi_{x, z}}(h, t)$. And, by (4.16), we obtain $0 \in S_{\varphi_{x, z}}(1, K t)$, which means $z \in S_{\varphi}(x, K t)$, and we prove the Theorem when $\varphi(y)=0$ and $\nabla \varphi(y)=0$.

The general case for $\varphi$ is treated as follows: given $y \in \mathbb{R}^{n}$, define the strictly convex auxiliary function $\varphi_{y}$ as

$$
\varphi_{y}(x)=\varphi(x)-\varphi(y)-\nabla \varphi(y)(x-y) \quad x \in \mathbb{R}^{n},
$$

then we have $\varphi_{y}(y)=0$ and $\nabla \varphi_{y}(y)=0$. Moreover, for every $x \in \mathbb{R}^{n}$ and $t>0$

$$
S_{\varphi_{y}}(x, t)=S_{\varphi}(x, t),
$$

and the theorem follows.

Corollary 5. $\varphi \in \operatorname{Eng}(n, K)$ if and only if for every $x, z \in \mathbb{R}^{n}, \varphi_{x, z} \in \operatorname{Eng}\left(1, K^{\prime}\right), K^{\prime}$ independent of $x$ and $z$.

Proof. The proof is clear from Lemma 3 and the proof of Theorem 4.

Given two objects $\mathscr{A}$ and $\mathscr{B}$ (numbers or functions), we shall write $\mathscr{A} \lesssim \mathscr{B}$ if there exists a constant $c$, depending only on $K$ (the engulfing constant), such that $\mathscr{A} \leqslant c \mathscr{B}$. If $\mathscr{A} \lesssim \mathscr{B}$ and $\mathscr{B} \lesssim \mathscr{A}$, we shall write $\mathscr{A} \simeq \mathscr{B}$. Thus, the condition on Theorem 4 reads

$$
\varphi(y)-\varphi(x)-\nabla \varphi(x) \cdot(y-x) \simeq(\nabla \varphi(x)-\nabla \varphi(y)) \cdot(x-y),
$$

for every $x, y \in \mathbb{R}^{n}$.

Corollary 6. Set $B(x, y)=\varphi(y)-\varphi(x)-\nabla \varphi(x) \cdot(y-x)$. If $\varphi \in \operatorname{Eng}(n, K)$, then the function $\delta_{\varphi}(x, y)=\max \{B(x, y), B(y, x)\}$ is a quasi-distance in $\mathbb{R}^{n}$ and $\delta_{\varphi} \simeq d_{\varphi}$.

Proof. The proof is immediate from Theorem 4. The function $B$ is known as the Bregman distance. Even if the Bregman distance is not a distance, under the presence of the engulfing property it becomes essentially a quasi-distance.

The following result relates the Euclidean balls and the $d_{\varphi}$-balls, providing the quantitative behaviour of $\varphi$. 
Theorem 7. Let $\varphi \in \operatorname{Eng}(n, K)$ and $r>0$. For $y \in \mathbb{R}^{n}$ define $\varphi_{y}(x)=\varphi(x)-\varphi(y)-$ $\nabla \varphi(y)(x-y)$. If $|x-y| \leqslant r$, then

$$
\begin{aligned}
\left(\min _{z:|z-y|=r} \varphi_{y}(z)\right)\left(\frac{|x-y|}{r}\right)^{1+K} & \leqslant \varphi(x)-\varphi(y)-\nabla \varphi(y)(x-y) \\
& \leqslant\left(\max _{z:|z-y|=r} \varphi_{y}(z)\right)\left(\frac{|x-y|}{r}\right)^{1+1 / K} .
\end{aligned}
$$

If $|x-y| \geqslant r>0$, then

$$
\begin{aligned}
\left(\min _{z:|z-y|=r} \varphi_{y}(z)\right)\left(\frac{|x-y|}{r}\right)^{1+1 / K} & \leqslant \varphi(x)-\varphi(y)-\nabla \varphi(y)(x-y) \\
& \leqslant\left(\max _{z:|z-y|=r} \varphi_{y}(z)\right)\left(\frac{|x-y|}{r}\right)^{1+K} .
\end{aligned}
$$

Proof. We shall first prove that if $\varphi \in \operatorname{Eng}_{0}(n, K)$ and $|x| \leqslant r$,

$$
\left(\min _{z:|z|=r} \varphi(z)\right)\left(\frac{|x|}{r}\right)^{1+K} \leqslant \varphi(x) \leqslant\left(\max _{z:|z|=r} \varphi(z)\right)\left(\frac{|x|}{r}\right)^{1+1 / K},
$$

and, if $|x| \geqslant r>0$, then

$$
\left(\min _{z:|z|=r} \varphi(z)\right)\left(\frac{|x|}{r}\right)^{1+1 / K} \leqslant \varphi(x) \leqslant\left(\max _{z:|z|=r} \varphi(z)\right)\left(\frac{|x|}{r}\right)^{1+K} .
$$

Consider first a function $\phi \in \operatorname{Eng}_{0}(1, K)$. By Theorem 4 we know that

$$
\frac{1}{K} \phi(t) \leqslant \phi^{\prime}(t) t-\phi(t) \leqslant K \phi(t)
$$

for every $t \in \mathbb{R}$. Let us work out the second inequality in the first place. For $t>0$, we get

$$
\frac{\phi^{\prime}(t)}{\phi(t)} \leqslant(1+K) \frac{1}{t}
$$

recognizing the derivatives of the corresponding logarithms, we get that the function $\phi(t) / t^{1+K}$ is decreasing in $(0, \infty)$. Now, given $x \in \mathbb{R}^{n}$, write $x=t x_{0}$, where $\left|x_{0}\right|=1$, and define $\phi(t)=\varphi\left(t x_{0}\right)$. By Lemma 3, $\phi \in \operatorname{Eng}_{0}(1, K)$. If $|x| \leqslant r$, then $t \leqslant r$ and we use the mentioned monotonicity to get

$$
\phi(r) / r^{1+K} \leqslant \phi(t) / t^{1+K}
$$

which is

$$
\varphi\left(r x_{0}\right) \frac{1}{r^{1+K}} \leqslant \varphi\left(t x_{0}\right) \frac{1}{t^{1+K}}=\varphi(x) \frac{1}{|x|^{1+K}}
$$

and the first inequality in (4.18) follows. The other inequalities are proven in similar fashion, by remarking that the function $\phi(t) / t^{1+1 / K}$ is increasing in $(0, \infty)$.

In order to finish the proof we need to consider the general case $\varphi \in \operatorname{Eng}(n, K)$. In this case, given $y \in \mathbb{R}^{n}$, define $\psi_{y}(x)=\varphi(x+y)-\varphi(y)-\nabla \varphi(y) \cdot x$. Thus, by 
Lemma 3, $\psi_{y} \in \operatorname{Eng}_{0}(n, K)$ and we complete the proof by applying (4.21) and (4.20) to the function $\psi_{y}$.

We have the following immediate consequence of Theorem 7

Corollary 8. Let $\varphi \in \operatorname{Eng}(n, K)$. For $y \in \mathbb{R}^{n}, \varphi_{y}$ defined as in Theorem 7 , and $r>0$,

$$
S_{\varphi}(y, m(\varphi, y, r)) \subset B(y, r) \subset S_{\varphi}(y, M(\varphi, y, r)),
$$

where $m(\varphi, y, r)=\min _{z:|z-y|=r} \varphi_{y}(z)$ and $M(\varphi, y, r)=\max _{z:|z-y|=r} \varphi_{y}(z)$.

\section{More properties of $\operatorname{Eng}(n)$. The convex conjugate}

As we saw, given $\varphi \in \operatorname{Eng}_{0}(n, K)$, the inequalities (4.22) imply that

$$
\varphi(x) \simeq \nabla \varphi(x) \cdot x
$$

(in particular, if $n=1$ the functions in $\operatorname{Eng}_{0}(1, K)$ verify the $\Delta_{2}$-condition), now we could ask if similar inequalities hold up to the second derivative, that is, is it true that $x D^{2} \varphi(x) x \simeq \varphi(x)$ (provided that $\varphi$ is twice differentiable)? As we will see, the answer is no.

Notice that $\operatorname{Eng}_{0}(n, K)$ is not contained in $C^{2}\left(\mathbb{R}^{n}\right)$ (take, for instance, $\varphi(x)=|x|^{p}$ with $2>p>1)$. To prove that the estimate $x D^{2} \varphi(x) x \simeq \varphi(x)$ does not hold in general, consider $n=1$ and pick a continuous doubling weight $w$ on $\mathbb{R}$ which vanishes at certain point $x_{0} \neq 0$. Set $\varphi(x) \doteq \int_{0}^{x} \int_{0}^{s} w(t) \mathrm{d} t \mathrm{~d} s \in \operatorname{Eng}_{0}(1, K)$ (see [7]), now we cannot have $w(x) x^{2} \simeq \varphi(x)$, since $\varphi$ is strictly positive when $x \neq 0$ and $w\left(x_{0}\right)=0$. However, an integral version of the inequalities $x D^{2} \varphi(x) x \simeq \varphi(x)$ does hold. More precisely, we have

Theorem 9. Let $\varphi \in \operatorname{Eng}(n, K) \cap C^{2}\left(\mathbb{R}^{n}\right)$. Then

$$
\varphi(x)-\varphi(y)-\nabla \varphi(y)(x-y) \simeq \int_{0}^{1} t(x-y) D^{2} \varphi(t x+(1-t) y) t(x-y) \mathrm{d} t .
$$

Proof. Consider first $\varphi \in \operatorname{Eng}_{0}(n, K)$, fix $x \in \mathbb{R}^{n}$ and define $f(t)=\varphi(t x) \in \operatorname{Eng}_{0}(1, K)$. As proved in [7], there exist positive constants $c_{K}, C_{K}$ depending only on $K$ such that

$$
c_{K} f(1) \leqslant \int_{0}^{1} t^{2} f^{\prime \prime}(t) \mathrm{d} t \leqslant C_{K} f(1)
$$

which yields

$$
c_{K} \varphi(x) \leqslant \int_{0}^{1} t x D^{2} \varphi(t x) t x \mathrm{~d} t \leqslant C_{K} \varphi(x) .
$$

To complete the proof, given any $\varphi \in \operatorname{Eng}(n, K)$, fix $y \in \mathbb{R}^{n}$ and define $\psi_{y}(x)=\varphi(x+$ $y)-\varphi(y)-\nabla \varphi(y) x \in \operatorname{Eng}_{0}(n, K)$ and apply (5.25) to $\psi_{y}$. 
We immediately have

Corollary 10. Let $\varphi \in \operatorname{Eng}(n, K) \cap C^{2}\left(\mathbb{R}^{n}\right)$. Then

$$
d_{\varphi}(x, y) \simeq \int_{0}^{1} t(x-y) D^{2} \varphi(t x+(1-t) y) t(x-y) \mathrm{d} t .
$$

Lemma 11. If $\varphi \in \operatorname{Eng}(n, K)$, then $\nabla \varphi: \mathbb{R}^{n} \rightarrow \mathbb{R}^{n}$ is a continuous bijection.

Proof. The continuity of $\nabla \varphi$ follows from Caffarelli's results mentioned in the Introduction. Injectivity of $\nabla \varphi$ follows from the strict convexity of $\varphi$. We could also use that $\varphi \in \operatorname{Eng}(n, K)$ to turn $\rho_{\varphi}$ into a quasi-distance, consequently

$$
\nabla \varphi(x)=\nabla \varphi(y) \Rightarrow \rho_{\varphi}(x, y)=0 \Rightarrow x=y .
$$

To prove that $\nabla \varphi$ is onto, note that it is enough to suppose $\varphi \in \operatorname{Eng}_{0}(n, K)$ ( $\operatorname{subtract}$ a hyperplane from $\varphi$ ). Thus, (4.21), with $r=1$, gives

$$
\lim _{|x| \rightarrow+\infty} \frac{\varphi(x)}{|x|}=+\infty
$$

Now, given $a \in \mathbb{R}^{n}$ we can minimize $h(x) \doteq \varphi(x)-a x$ to get that $a \in \nabla \varphi\left(\mathbb{R}^{n}\right)$.

Theorem 12. Let $\varphi$ be in $\operatorname{Eng}(n, K)$. If $\varphi^{*}$ denotes the conjugate of $\varphi$, then $\varphi^{*} \in$ $\operatorname{Eng}\left(n, K^{*}\right)$ with $K^{*}$ depending only on $K$. Moreover, the sections of $\varphi$ and $\varphi^{*}$ are related as follows: for every $x \in \mathbb{R}^{n}, t>0$

$$
\nabla \varphi\left(S_{\varphi}(x, t / K)\right) \subset S_{\varphi^{*}}(\nabla \varphi(x), t) \subset \nabla \varphi\left(S_{\varphi}(x, K t)\right) .
$$

Proof. Recall that

$$
\varphi^{*}(x)=\sup _{z \in \mathbb{R}^{n}}(x z-\varphi(z)) .
$$

Since $\varphi$ has the engulfing property, we know that $\varphi$ is a strictly convex differentiable function. By Theorem 26.5 in [10], we get that $\varphi^{*}$ is also a strictly convex differentiable function whose domain is $\nabla \varphi\left(\mathbb{R}^{n}\right)$ which, by Lemma 11 , equals $\mathbb{R}^{n}$. We also have

$$
\nabla \varphi\left(\nabla \varphi^{*}(x)\right)=\nabla \varphi^{*}(\nabla \varphi(x))=x \quad \forall x \in \mathbb{R}^{n}
$$

and

$$
\varphi^{*}(\nabla \varphi(x))=\nabla \varphi(x) x-\varphi(x) \quad \forall x \in \mathbb{R}^{n},
$$

(remark that (5.29) and (4.22) imply $\left.\varphi^{*}(\nabla \varphi(x)) \simeq \varphi(x)\right)$. Moreover, $\left(\varphi^{*}\right)^{*}=\varphi$. We first note that for every $x, y \in \mathbb{R}^{n}$

$$
y \in S_{\varphi}(x, t) \Leftrightarrow \nabla \varphi(x) \in S_{\varphi^{*}}(\nabla \varphi(y), t) .
$$

To prove (5.30), we do as follows: $y \in S_{\varphi}(x, t)$ if and only if

$$
\varphi(y)<\varphi(x)+\nabla \varphi(x)(y-x)+t=\varphi(x)-\nabla \varphi(x) x+\nabla \varphi(x) y+t
$$


Now, we use (5.29) to get the equivalent condition

$$
\varphi(y) y-\varphi^{*}(\nabla \varphi(y))<-\varphi^{*}(\nabla \varphi(x))+\nabla \varphi(x) y+t
$$

which is the same as

$$
\varphi^{*}(\nabla \varphi(x))<\varphi^{*}(\nabla \varphi(y))+y \cdot(\nabla \varphi(x)-\nabla \varphi(y))+t
$$

and, by (5.28), this means $\nabla \varphi(x) \in S_{\varphi^{*}}(\nabla \varphi(y), t)$. Thus, (5.30) and (ii) in Theorem 4 imply that $\varphi^{*} \in \operatorname{Eng}\left(n, K^{*}\right)$, for some $K^{*}$ depending only on $K$. Following up the constants we can take $K^{*}=2 K(K+1)$.

The next step is to prove the following inclusions for every $x \in \mathbb{R}^{n}, t>0$ :

$$
\nabla \varphi\left(S_{\varphi}(x, t)\right) \subset S_{\varphi^{*}}(\nabla \varphi(x), K t) \subset \nabla \varphi\left(S_{\varphi}\left(x, K^{2} t\right)\right) .
$$

To prove the first inclusion, let us take $z \in \nabla \varphi\left(S_{\varphi}(x, t)\right)$. Then, $z=\nabla \varphi(y)$ for some $y \in S_{\varphi}(x, t)$; and, by the engulfing property for $\varphi, x \in S_{\varphi}(y, K t)$. Now, by (5.30), $z=\nabla \varphi(y) \in S_{\varphi^{*}}(\nabla \varphi(x), K t)$.

To prove the second inclusion, take $z \in S_{\varphi^{*}}(\nabla \varphi(x), K t)$. By (5.28), $z=\nabla \varphi(y)$ for some $y \in \mathbb{R}^{n}$. Then $\nabla \varphi(y) \in S_{\varphi^{*}}(\nabla \varphi(x), K t)$, and by using (5.30) we get $x \in S_{\varphi}(y, K t)$. Again by the engulfing property, $y \in S_{\varphi}\left(x, K^{2} t\right)$, which implies $z \in \nabla \varphi\left(S_{\varphi}\left(x, K^{2}, t\right)\right)$. Applying $\nabla \varphi^{*}$ in (5.31), we obtain

$$
S_{\varphi}(x, t) \subset \nabla \varphi^{*}\left(S_{\varphi^{*}}(\nabla \varphi(x), K t)\right) \subset S_{\varphi}\left(x, K^{2} t\right) .
$$

Corollary 13. If $\varphi \in \operatorname{Eng}(n)$, then $\nabla \varphi: \mathbb{R}^{n} \rightarrow \mathbb{R}^{n}$ is a homeomorphism.

Proof. Immediate from Lemma 11 and Theorem 12, since the continuous inverse of $\nabla \varphi$ is $\nabla \varphi^{*}$.

Corollary 14. If $\varphi, \psi \in \operatorname{Eng}(n)$, then the infimal convolution $\varphi \odot \psi \in \operatorname{Eng}(n)$.

Proof. Recall that the infimal convolution of two convex functions $\varphi$ and $\psi$ is the convex function defined by

$$
\varphi \odot \psi(x)=\inf _{y \in \mathbb{R}^{n}}\{\varphi(y)-\psi(x-y)\},
$$

and we always have $(\varphi \odot \psi)^{*}=\varphi^{*}+\psi^{*}$. Thus, we get the result applying Lemma 3 and Theorem 12.

\section{A constructive approach to Caffarelli's $C^{1, \beta}$ regularity result}

As mentioned in the Introduction, Caffarelli proved the $C^{1, \beta}$ regularity of any convex function $\varphi \in \operatorname{Eng}(n, K)$. His proof is based on a compactness argument that does not provide an estimate for $\beta$ or the $C^{1, \beta}$ norm of $\varphi$ on compact sets. The task of finding the explicit size of these constants was posed as an open problem in Villani's recent book (see [11, p. 141]). 
In this section we will get such estimates, in terms of $K$, through Theorem 7 . To illustrate the main idea, let us take a look at the case $n=1$. Consider $\varphi \in \operatorname{Eng}_{0}(1, K),|x| \leqslant 1$, and denote by $M(\varphi, 1)$ the maximum between $\varphi(1)$ and $\varphi(-1)$. Then, by (4.18), we get $\varphi(x) \leqslant M(\varphi, 1)|x|^{1+1 / K}$. On the other hand, by (4.22), we have $0 \leqslant \varphi^{\prime}(x) x \leqslant(K+$ 1) $\varphi(x)$. Consequently, for every $x$ with $|x| \leqslant 1$, we get $\left|\varphi^{\prime}(x)\right| \leqslant(K+1) M(\varphi, 1)|x|^{1 / K}$. Which is the $C^{1 / K}$ regularity of $\varphi^{\prime}$ about 0 . Before stating the general result some notation is in order. Given a convex function $\phi \in \operatorname{Eng}(n, K), y \in \mathbb{R}^{n}$, and $r>0$, set

$$
M(\phi, y, r)=\max _{z:|z-y|=r}\{\phi(z)-\phi(y)-\nabla \phi(y) \cdot(z-y)\}
$$

and

$$
m(\phi, y, r)=\min _{z:|z-y|=r}\{\phi(z)-\phi(y)-\nabla \phi(y) \cdot(z-y)\}
$$

Theorem 15. Let $\varphi \in \operatorname{Eng}(n, K), \varphi^{*} \in \operatorname{Eng}\left(n, K^{*}\right)$, and $y \in \mathbb{R}^{n}$. For every $z \in \mathbb{R}^{n}$ with $|z-y| \leqslant r$, we have

$$
\frac{|\nabla \varphi(z)-\nabla \varphi(y)|}{|z-y|^{1 / 1+K^{*}}} \leqslant C\left(r, K, m\left(\psi_{y}^{*}, 0,1\right), M(\varphi, y, r)\right)
$$

where $\psi_{y}^{*}$ is the convex conjugate to

$$
\psi_{y}(x)=\varphi(x+y)-\varphi(y)-\nabla \varphi(y) \cdot x
$$

Proof. As usual, let us begin considering the case $\varphi \in \operatorname{Eng}_{0}(n, K)$ and $y=0$. Take $x$ with $|x| \leqslant r$, by (4.18) we get

$$
\varphi(x) \leqslant M(\varphi, 0, r)\left(\frac{|x|}{r}\right)^{1+1 / K} \leqslant M(\varphi, 0, r) .
$$

Next, observe that if $|\nabla \varphi(x)| \geqslant 1$, then, by (4.19) applied to $\varphi^{*}$,

$$
m\left(\varphi^{*}, 0,1\right)|\nabla \varphi(x)|^{1+1 / K^{*}} \leqslant \varphi^{*}(\nabla \varphi(x)) \leqslant K \varphi(x) \leqslant K M(\varphi, 0, r),
$$

where we used (5.29) and Theorem 4 to write $\varphi^{*}(\nabla \varphi(x))=\nabla \varphi(x) x-\varphi(x) \leqslant K \varphi(x)$. All this gives,

$$
|\nabla \varphi(x)| \leqslant \max \left\{1,\left(\frac{K M(\varphi, 0, r)}{m\left(\varphi^{*}, 0,1\right)}\right)^{K^{*} / K^{*}+1}\right\} \doteq C_{1}=C_{1}(\varphi, r, K) .
$$

Now we can apply (4.18) to $\varphi^{*}$ with $C_{1}$ and at $\nabla \varphi(x)$ to get

$$
m\left(\varphi^{*}, 0, C_{1}\right)\left(\frac{|\nabla \varphi(x)|}{C_{1}}\right)^{1+K^{*}} \leqslant \varphi^{*}(\nabla \varphi(x)) \leqslant K \varphi(x)
$$

that is,

$$
|\nabla \varphi(x)| \leqslant C_{1}\left(\frac{K}{m\left(\varphi^{*}, 0, C_{1}\right)}\right)^{1 / 1+K^{*}} \varphi(x)^{1 / 1+K^{*}}
$$


and dividing by $|x|^{1 / 1+K^{*}}$,

$$
\frac{|\nabla \varphi(x)|}{|x|^{1 / 1+K^{*}}} \leqslant C_{1}\left(\frac{K}{m\left(\varphi^{*}, 0, C_{1}\right)}\right)^{1 / 1+K^{*}}\left(\frac{\varphi(x)}{|x|}\right)^{1 / 1+K^{*}} .
$$

Note that $\varphi(x) /|x|=\varphi(|x| x /|x|) /|x|$ and for any $z \in \mathbb{R}^{n}$ the function $t \rightarrow \varphi(t z) / t$ is increasing. Therefore, since $|x| \leqslant r$, we get

$$
\begin{aligned}
\frac{|\nabla \varphi(x)|}{|x|^{1 / 1+K^{*}}} & \leqslant C_{1}\left(\frac{K}{m\left(\varphi^{*}, 0, C_{1}\right)}\right)^{1 / 1+K^{*}}\left(\frac{\varphi(r x /|x|)}{r}\right)^{1 / 1+K^{*}} \\
& \leqslant C_{1}\left(\frac{K}{m\left(\varphi^{*}, 0, C_{1}\right)}\right)^{1 / 1+K^{*}}\left(\frac{M(\varphi, 0, r)}{r}\right)^{1 / 1+K^{*}} .
\end{aligned}
$$

To complete the proof, given $\varphi \in \operatorname{Eng}(n, K)$ and $y \in \mathbb{R}^{n}$, set $\psi_{y}(x)=\varphi(x+y)-\varphi(y)-$ $\nabla \varphi(y) x \in \operatorname{Eng}_{0}(n, K)$ and $z=x+y$ to get, for $|z-y| \leqslant r$,

$$
\frac{|\nabla \varphi(z)-\nabla \varphi(y)|}{|z-y|^{1 / 1+K^{*}}} \leqslant C_{y}\left(\frac{K}{m\left(\psi_{y}^{*}, 0, C_{y}\right)}\right)^{1 / 1+K^{*}}\left(\frac{M(\varphi, y, r)}{r}\right)^{1 / 1+K^{*}}
$$

where

$$
C_{y} \doteq \max \left\{1,\left(\frac{K M(\varphi, y, r)}{m\left(\psi_{y}^{*}, 0,1\right)}\right)^{K^{*} / 1+K^{*}}\right\} .
$$

Thus, $\nabla \varphi$ is in $C^{\beta}$ with $\beta=1 / 1+K^{*}$ and $K^{*}=2 K(K+1)$.

\section{Further remarks}

If the Monge-Ampère measure $\mu_{\varphi}$ satisfies the (DC)-doubling condition with constants $C$ and $\alpha$, then $\varphi \in \operatorname{Eng}(n, K)$ with

$$
K=\frac{2^{n+2} w_{n} w_{n-1}}{\alpha_{n}^{n+1}} \frac{C}{(1-\alpha)^{n}}+1,
$$

where $w_{k}$ is the volume of the $k$-dimensional unit ball and $\alpha_{n}=n^{-3 / 2}$. In the case $\lambda \leqslant \operatorname{det} D^{2} \varphi \leqslant \Lambda$, if we set $\alpha=1 / 2$ we get $C=2^{n} \Lambda / \lambda$. These constants can be easily followed up from [8].

Although we consider solutions to $\operatorname{det} D^{2} \varphi=\mu$ in $\mathbb{R}^{n}$, the main results in this paper can be proved (after slight modifications) for solutions to the Monge-Ampère equation in a bounded convex domain $\Omega \subset \mathbb{R}^{n}$.

\section{References}

[1] H. Aimar, L. Forzani, R. Toledano, Balls and quasi-metrics: a space of homogeneous type modeling the real analysis related to the Monge-Ampère equation, J. Fourier Anal. Appl. 4 (1998) 377-381.

[2] L. Caffarelli, Some regularity properties of solutions of Monge-Ampère equation, Comm. Pure Appl. Math. 44 (1991) 965-969. 
[3] L. Caffarelli, Boundary regularity of maps with convex potentials, Comm. Pure Appl. Math. 45 (1992) 1141-1151.

[4] L. Caffarelli, C. Gutiérrez, Real analysis related to the Monge-Ampère equation, Trans. Am. Math. Soc. 348 (1996) 1075-1092.

[5] L. Caffarelli, C. Gutiérrez, Properties of the solutions of the linearized Monge-Ampère equation, Am. J. Math. 119 (2) (1997) 423-465.

[6] L. Forzani, D. Maldonado, On geometric characterizations for Monge-Ampère doubling measures, J. Math. Anal. Appl. 275 (2) (2002) 721-732.

[7] L. Forzani, D. Maldonado, Doubling weights, quasi-symmetric mappings, and a class of convex functions on the real line, submitted for publication.

[8] C. Gutiérrez, The Monge-Ampère Equation. Progress in Nonlinear Differential Equations and their Applications, Vol. 44, Birkhäuser, 2001.

[9] C. Gutiérrez, Q. Huang, Geometric properties of the sections of solutions to the Monge-Ampère equation, Trans. Am. Math. Soc. 352 (9) (2000) 4381-4396.

[10] R.T. Rockafellar, Convex Analysis, Princeton University Press, Princeton, NJ, 1970.

[11] C. Villani, Topics in Optimal Transportation, Graduate Studies in Mathematics, American Mathematical Society, Vol. 58, 2003. 